\title{
SUMMARY
}

The aim of this report is to demonstrate that the biogeochemical approach provides a uniting framework for managing the environment of our planet and in particular the environment of a planet reshaped by Man in the best possible way.

This framework is based on the study of the biogeochemical cycles that characterize the biosphere (i.e. the place where life is present) and that are naturally linked to the Earth's overall geochemical cycles.

The above considerations lead us to define the limits of this report.

1. This report does not take into account all of the planet, only its upper surface which is where external geodynamical phenomena occur and where, in certain situations, some internal geodynamical phenomena (for instance, volcanism) may act.

2. Regarding this upper surface, the report focuses more particularly on the continental areas. Emerged land and oceans are of course linked, however because they do not function in a similar fashion at the surface of the planet, the report mainly addresses terrestrial ecosystems.

3. Regarding the continents, the report focuses on the biosphere, which constitutes the ecological system.

This ecological system is situated between:

- the climatic system, where the oceanic and atmospheric cycle is primordial;

- the geological system, which corresponds to the geosphere devoid of animals.

4. Within the terrestrial ecological system, the circulation of elements involves four kinds of relations:

- plant-atmosphere: the atmospheric sub-cycle;

- internal to plants: the biochemical sub-cycle;

- soil-plant: the biological sub-cycle;

- rock-soil: the geochemical sub-cycle. 
Biogeochemical cycles rely mainly on the last two sub-cycles which both involve soils, hence they are pivotal to the operation of the terrestrial biosphere.

The goal of this report is not to describe the biogeochemical cycles of all the chemical elements, but to show why and how these cycles have a significant role in the evolution of a planet shaped by man.

In order to do so, the report is divided into two units and four parts:

In the first unit, all the information that is directly linked to understanding geochemical cycles is brought together. It is divided in two parts.

The first part concerns the description of the biogeochemical cycle of some of the elements that play a major role in the biogeosphere. We have focused on:

- carbon, because it is involved in all of the cycles, i.e. the atmospheric, ecological and geological cycles (chapter 1);

- nitrogen, phosphorus and sulfur because they are specific to the living world and because their role is likely to be primordial in the environment (chapter 2);

- silicon, aluminum and iron because they allow us to make a bridge between the ecological and the geological systems (chapter 3);

- finally, radionuclides (natural and artificial), due to their impact on the biological environment (chapter 4);

The second part concerns the biogeochemical study of a number of representative environments of the natural and man-shaped planet.

Regarding natural ecosystems, we have focused on:

- forest ecosystems, which are highly characteristic of terrestrial environments and which are the site of offen very efficient biogeochemical recycling (chapter 5.1);

- oceanic environments. Although not part of our topic, the biogeochemistry of these environments is described very briefly to show that in this field everything at the surface of the planet is linked (chapter 5.2).

In environments that are highly influenced by Man where the excess and overload of certain elements can lead to dysfunctions, we have focused on three types of environments: 
- the agricultural sector, where we looked at the practice of manuring using exogenous organic matter (EOM) which is found in areas of intensive breeding farms (chapter 6.1);

- the industrial sector; we present a number of environments where certain elements are in excess, such as can happen in mining and metallurgy areas (chapter 6.2);

- Finally, we looked at estuary environments because they are the collection point for numerous contaminants coming from the continents (chapter 6.3).

The second unit proceeds from all we have learnt in the first, briefly that biogeochemical cycles are the consequence of complex systems that necessitate many scientific fields and numerous data. This consideration implies:

a) further studies in certain scientific areas;

b) a particular interest in data collection;

c) the reliance on model building.

Point a) constitutes the third part which stresses the development of all venues concerning the role of the soil as a living environment. Knowledge is still lacking in this area. Concerning this issue, two points are considered in particular:

- research on micro-organisms that are crucial for transforming waste and other residues from living beings and their return to the mineral state (chapter 7);

- research on soil organic matter, which plays a fundamental role because it is an intermediate between the mineral world and the living world (chapter 8$)$.

Points b) and c) are treated in the fourth part of the report which discusses model operations (chapter 9) and problems linked to a generalized collection of data (for instance, observatories, networks) (chapter 10).

\section{Conclusions and recommendations}

Our conclusions and recommendations concern more specifically the following three points:

- to support in the future a number of disciplines which are still orphan, for example bioinorganic chemistry, microbial ecology, soil science; 
- to go beyond the classic disciplines in this area of research and emphasize multidisciplinarity. This implies:

- creating transversal departments within the major research organizations;

- the development of relations between organizations to create mixed research units;

- supporting actions in the area of observatories: creation, follow-up and mostly long-term maintenance;

- finally, regarding higher education, to contemplate the creation of university structures that make it possible to train qualified students in this sector which is crucial to the future of humanity. 